\title{
Intuitionistic fuzzy optimization: Usage of hesitation index
}

\author{
Arindam Garai, Tapan Kumar Roy \\ Department of Mathematics, Sonarpur Mahavidyalaya, Sahid Biswanath Sarani, Sonarpur, Kolkata-149 \\ fuzzy_arindam@yahoo.com
}

Department of Mathematics, Bengal Engineering and Science University, Shibpur, Howrah-103

roy_t_k@yahoo.co.in

\begin{abstract}
This paper presents the concept of usage of hesitation index in optimization problem under uncertainty. Our technique is an extension of idea of intuitionistic fuzzy optimization technique, proposed by Plamen P. Angelov in 1997, which is widely considered as a successful intuitionistic fuzzy optimization tool by researchers all over the world. It is well known that the advantages of the intuitionistic fuzzy optimization problems are twofold: firstly, they give the richest apparatus for formulation of optimization problems and on the other hand, the solution of intuitionistic fuzzy optimization problems can satisfy the objective(s) with bigger degree of satisfaction than the analogous fuzzy optimization problem and the crisp one. Angelov's approach is an application of the intuitionistic fuzzy (IF) set concept to optimization problems. In his approach, the degree of acceptance is maximized while the degree of rejection is minimized. In our approach, not only the degree of acceptance is maximized and the degree of rejection is minimized but also the degree of hesitation is minimized. For the sake simplicity alone, the same problem, as studied by Angelov, is considered. Varied importance (and hence weights) to each of the degree of acceptance and the degree of rejection and the degree of hesitation have been given. Tables with these results are formulated and compared among.
\end{abstract}

\section{Indexing terms/Keywords}

Fuzzy Optimization; Intuitionistic Fuzzy Optimization; Hesitation Index; Weight.

\section{Academic Discipline And Sub-Disciplines}

Mathematics; Computer Science; Operation Research;

\section{SUBJECT CLASSIFICATION}

AMS Classification: Fuzzy Programming 90C70; Decision Theory and Fuzziness 62C86; Optimization and Variational Techniques 65K10;

\section{TYPE (METHOD/APPROACH)}

Intuitionistic Fuzzy Optimization Technique Analysis using Hesitation Index.

\section{Council for Innovative Research}

\author{
Peer Review Research Publishing System
}

Journal: INTERNATIONAL JOURNAL OF COMPUTERS \& TECHNOLOGY

Vol 10, No 4

editor@cirworld.com

www.cirworld.com, member.cirworld.com 


\section{INTRODUCTION}

As it is already mentioned by Angelov in his historic paper in 1997 [1], deterministic optimization problems are well studied, but they are very limited and in many cases they do not represent exactly the real problem. Usually, it is difficult to describe the constraints of an optimization problem by strict crisp relations (equalities and/or non-equalities). Practically, a small violation of a given constraint is admissible and it can lead to a more efficient solution of the real problem.

In over the last three decades, optimization problems have been investigated in the sense of fuzzy set theory. Fuzzy optimization formulations are more flexible and allow finding solutions which are more adequate to the real problem in comparison with crisp problems.

Again, fuzzy set theory has been widely developed and various modifications and generalizations have appeared. One of them is the concept of intuitionistic fuzzy (IF) sets [2, 3, 4]. They consider not only the degree of membership to a given set, but also the degree of rejection such that the sum of both values is less than or equal to one [8]. Applying this concept Angelov [1] has reformulated the optimization problem.

Angelov [1] has well identified that, in general, an optimization problem includes objective(s) and constraint(s). In fuzzy optimization problems, the objective(s) and/or constraint(s) or parameter(s) and relation(s) are represented by fuzzy sets. The solution of crisp optimization problem must satisfy all constraints exactly. And in the analogous fuzzy optimization problem, the degree of satisfaction of objective(s) and of constraint(s) is maximized. It is transformed via Bellman-Zadeh's approach [5].

In the case when the degree of rejection (non-membership) is defined simultaneously with the degree of acceptance (membership) and when both these degrees are not complementary to each other, IF sets can be used as a more general and complete tool for describing this uncertainty.

Yet, in Angelov's paper [1], the degree of hesitation is not used at all. It must be noted that Szmidt and Kacprzyk [7] have already mentioned, in 2000, that taking into account the third parameter (degree of hesitation) when calculating the Euclidean distance for intuitionistic fuzzy sets does have an influence on the final result. It should be so because a two dimensional geometric interpretation is an orthogonal projection of a real situation as shown suitably in their historic paper.

It is also worth observing that the importance and hence weight in maximizing the degree of acceptance and in minimizing the degree of rejection is equal in Angelov's paper [1]. But, in real life situation, a good decision is one in which the decision maker is more certain and less hesitant. Hence in our decision making process, reducing the degree of hesitation is more important than maximizing the degree of acceptance or minimizing the degree of rejection. Having in mind the above mentioned facts, in order to be more concordant with the mathematical notion, we have used this third parameter with greater importance [9] in our study. Next, we have used different importance level to each of these three parameters and compared the result in a table and studied it.

\section{DEFINITION}

\section{Fuzzy Set}

A fuzzy subset $\tilde{A}$ of $X$ is defined by its membership function $\mu_{\tilde{A}}: X \rightarrow[0,1]$, that assigns to every $x \in X$, a real number $\mu_{\tilde{A}}(x)$ in the closed unit interval $[0,1]$, where the value of $\mu \tilde{A}(x)$ at $x$ represents the grade of membership of $x$ in $\tilde{A}$. So nearer the value of $\mu_{\tilde{A}}(x)$ is unity, the grade of membership of $x$ in $\tilde{A}$. A fuzzy subset $\tilde{A}$ of $X$ can be characterized as a set of ordered pair of elements $x$ and its grade $\mu \tilde{A}(x)$ and is often written as $\tilde{A}=\{(x, \mu \tilde{A}(x)): x \in X\}$. When the membership function $\mu \tilde{A}(x)$ contains only two points 0 and 1 , membership function $\mu_{\tilde{A}}(x)$ is identical to the characteristic function $\chi: X \rightarrow[0,1]$ and in that case $\tilde{A}$ is a crisp set.

\section{Intuitionistic Fuzzy Set}

On the other hand, an intuitionistic fuzzy set $A$ in $X$ is defined by $A=\left\{<x ; \mu_{A}(x), v_{A}(x)>\mid x \in E\right\}$ where $\mu_{A}: E \rightarrow[0,1]$ and $v_{A}$ : $E \rightarrow[0,1]$ with the condition $0 \leq\left(\mu_{A}(x)+v_{A}(x)\right) \leq 1$, where $\mu_{A}(x)$ and $v_{A}(x)$ denote the degree of membership and non membership respectively [2]. It is clear that for every fuzzy set $A$, there exist an intuitionistic fuzzy set $A=\left\{<x ; \mu_{A}(x), 1-\right.$ $\left.\mu_{A}(x)>\mid x \in E\right\}$. Also, for each intuitionistic fuzzy set in $X$, we set $\pi_{A}(x)=1-\mu_{A}(x)-v_{A}(x)$ and it is called the degree of hesitancy or hesitation index or intuitionistic index of $x$ in $A$. It is clear that $0 \leq \pi_{A}(x) \leq 1$ for each $x$ in universal set $[2,3,4]$.

\section{INTUITIONISTIC FUZZY OPTIMIZATION PROBLEM}

Let us consider a crisp optimization problem of the form:

$$
\begin{aligned}
& \text { Minimize } f_{i}(x), \quad i=1 \ldots p, \\
& \text { Subject to constraints } g_{j}(x) \leq 0, j=1 \ldots q,
\end{aligned}
$$

where $x$ denotes the unknown variables, $f_{i}(x)$ are the objective functions, $g_{j}(x)$ are the constraints (non-equalities), $p$ denotes the number of objectives and $q$ denotes the number of constraints. The solution of this crisp optimization problem 
satisfies all constraints exactly. In the analogous fuzzy optimization problem, the degree of satisfaction of objective(s) as well as of constraint(s) is maximized. The problem is of the form

$$
\begin{aligned}
& \text { Minimize } \mathrm{f}_{\mathrm{i}}(\mathrm{x}), \quad \mathrm{i}=1 \ldots \mathrm{p}, \\
& \text { Subject to constraints } \mathrm{g}_{\mathrm{j}}(\mathrm{x}) \simeq 0, \quad \mathrm{j}=1 \ldots \mathrm{q},
\end{aligned}
$$

where Minimize denotes the fuzzy minimization and $\tilde{\leq}$ denotes the fuzzy inequality. Next, it is transformed via BellmanZadeh's approach [5] to the following optimization problem: To maximize the degree of membership (acceptance) of the objective(s) as well as of the constraints to the respective fuzzy sets:

$$
\text { Maximize } \mu_{\mathrm{i}}(\mathrm{x}), \quad x \in R^{n}, i=1 \ldots(p+q),
$$

Subject to constraints $0 \leq \mu_{\mathrm{i}}(\mathrm{x}) \leq 1$,

where $\mu_{\mathrm{i}}(\mathrm{x})$ denotes degree of acceptance of $\mathrm{x}$ in $R^{n}$.Again, according to Angelov's famous paper in 1997 on IFO technique [6], when the degree of rejection (non-acceptance) is defined simultaneously with the degree of acceptance (membership) and when both these degrees are not complementary to each other, intuitionistic fuzzy set [3, 4] can be used as a more general tool for describing this fuzziness. It is possible to represent deeply existing nuances in problem formulation defining objectives and constraints (or part of them) by IF sets i.e. a pairs of membership functions $\mu$ s (xij) (of sth IF sets) and non-membership functions vs (xij) (of sth IF sets). So, according to Angelov [1], to maximize the degree of acceptance of IF objectives and constraints and to minimize the degree of rejection of IF objectives and constraints, we have the following:

$$
\begin{array}{ll}
\text { Maximize } & \mu_{\mathrm{i}}(x), i=1 \ldots(p+q), \\
\text { Minimize } & \mathrm{v}_{\mathrm{i}}(x), i=1 \ldots(p+q),
\end{array}
$$

Subject to constraints

$$
\begin{array}{ll}
v_{i}(x) \geq 0, & i=1 \ldots(p+q) \\
\mu_{i}(x) \geq v_{i}(x), & i=1 \ldots(p+q), \\
\mu_{i}(x)+v_{i}(x) \leq 1, & i=1 \ldots(p+q) .
\end{array}
$$

Where $\mu_{i}(x)$ denotes the degree of membership of $x$ to the $i^{\text {th }}$ IF set and $v_{i}(x)$ denotes the degree of rejection of $x$ to the $i^{\text {th }}$ IF set.

\section{USAGE OF HESITATION INDEX IN PROBLEM FORMULATION}

It may be noted that the degree of hesitation $\pi=(1-\mu-v)$ is never used in the above mentioned procedure. As already explained, in 2000, Szmidt and Kacprzyk [7] have already mentioned that taking into account the third parameter (degree of hesitation) when calculating the Euclidean distance for intuitionistic fuzzy sets does have a clear influence on the final result. Also the output is consistent as desired. Moreover, in a decision making process it is desired that there will be minimal hesitation in the final decision. The final outcome may be either acceptable or not acceptable but if it has a bigger degree of hesitancy; it will be difficult for the decision maker to come to a well acceptable conclusion. Hence, we plan to minimize the degree of hesitation as well.

In Angelov's paper [1], it is also noted that the degree of acceptance and degree of rejection have same importance in finding optimum solution. So initially, we may provide same importance to both of them while providing more importance in minimizing the degree of hesitation. So, to maximize the degree of acceptance of IF objectives and constraints and to minimize the degree of rejection of IF objectives and constraints (providing same weightage) and to minimize the degree of hesitation of IF objectives and constraints (with higher weightage), the following problem needs to be solved:

$$
\begin{array}{ll}
\text { Maximize } & w_{1} \cdot \mu_{i}(x), i=1 \ldots(p+q), \\
\text { Minimize } & w_{1} \cdot v_{i}(x), i=1 \ldots(p+q), \\
\text { Minimize } & w_{2} \cdot\left(1-\mu_{i}(x)-v_{i}(x)\right), i=1 \ldots(p+q),
\end{array}
$$

Subject to the constraints

$$
\begin{aligned}
& \mu_{i}(x) \geq v_{i}(x), i=1 \ldots(p+q), \\
& \mu_{i}(x)+v_{i}(x) \leq 1, i=1 \ldots(p+q) \\
& v_{i}(x) \geq 0, i=1 \ldots(p+q),
\end{aligned}
$$

where $\mathrm{w}_{1}$ and $\mathrm{w}_{2}$ are weights such that $\mathrm{w}_{1}+\mathrm{w}_{1}+\mathrm{w}_{2}=1$. It may be recalled that $\pi_{i}=1-\mu_{i}-v_{i} \forall i=1 \ldots(p+q)$. 


\section{NUMERICAL EXAMPLE OF INTUITIONISTIC FUZZY OPTIMIZATION PROBLEM}

We shall consider the same transportation problem that was considered by Angelov [1] in 1997. It states that costs of a delivery from the $\mathrm{i}^{\text {th }}$ port to the $\mathrm{j}^{\text {th }}$ market (in thousands of dollars) are given as in the respective cells of Table 1.

Table 1. Cost of delivery from $\mathrm{i}^{\text {th }}$ port to $\mathrm{j}^{\text {th }}$ market

\begin{tabular}{|c|c|c|c|c|c|}
\hline & Market 1 & Market 2 & Market 3 & Market 4 & Capacity \\
\hline Port 1 & 10 & 7 & 4 & 1 & 400 \\
\hline Port 2 & 2 & 7 & 10 & 6 & 150 \\
\hline Port 3 & 8 & 5 & 3 & 2 & 350 \\
\hline Demand & 200 & 200 & 100 & 350 & \\
\hline
\end{tabular}

The demands of loads in every market and the capacity of loads in every port are given (in tons) in the last column (row) of Table 1. An optimal transportation plan $\mathrm{x}\left(\mathrm{x} \in R^{n}\right)$ which minimizes the cost has to be determined. Practically, the demands of markets are determined on the basis of sales forecasting. If the prognosis for Market 4 is about 350 (t), the following IF set, according to Angelov, seems to be a more realistic description:

$$
\mu_{4}=\frac{1}{1+0.01\left(x_{14}+x_{24}+x_{34}-350\right)^{2}}, \quad v_{4}=\frac{\left(x_{14}+x_{24}+x_{34}-350\right)^{2}}{500+\left(x_{14}+x_{24}+x_{34}-350\right)^{2}} .
$$

It means that the degree of rejection $\left(v_{4}\right)$ is also defined which determines the worst admissible case. In general, it cannot simply be a complement to the degree of acceptance. The degree of acceptance $\left(\mu_{4}\right)$ of values of the demand in Market 4 increases more rapidly than the rejection $\left(v_{4}\right)$ decreases such that their sum is less than 1 . By analogy, the rest of the IF sets can be defined. Three of them determine the demand of Market 1, Market 2 and Market 3 and the other three sets determine the capacity of Port 1, Port 2 and Port 3, taking into account the subjective estimation of acceptance of various values of the demand and the capacity. It is supposed that the prognosis for Market 2 is about 200 (t). Thus, it may be assumed that

$$
\mu_{2}=\frac{1}{1+0.01\left(x_{12}+x_{22}+x_{32}-200\right)^{2}}, \quad v_{2}=\frac{\left(x_{12}+x_{22}+x_{32}-200\right)^{2}}{500+\left(x_{12}+x_{22}+x_{32}-200\right)^{2}} .
$$

Angelov assumed that the demand of Market 1 and Market 3 and the capacity of all ports are defined by crisp sets [1]. The IF objective can be determined by degrees of acceptance $\left(\mu_{0}\right)$ and rejection $\left(v_{0}\right)$ of the cost function as follows:

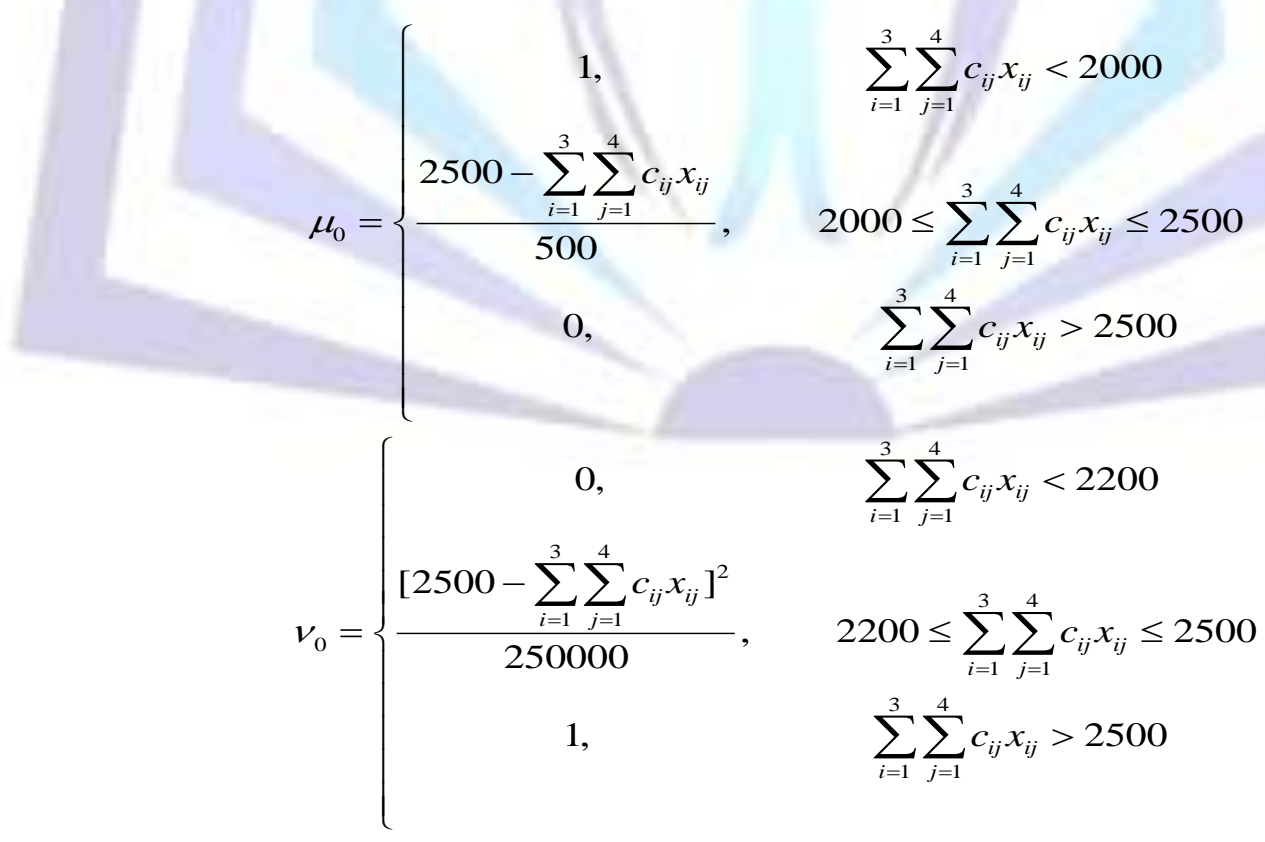

where $C$ is given by $C=\left|\begin{array}{cccc}10 & 7 & 4 & 1 \\ 2 & 7 & 10 & 6 \\ 8 & 5 & 3 & 2\end{array}\right|$. 
It is possible that a part of constraints and objective(s) are intuitionistic fuzzy and others are fuzzy or crisp. The problem is thus formulated (while using hesitation index) as follows:

$$
\begin{aligned}
& \text { Maximize } \mathrm{w}_{1}\left\{\mu_{0}(x), \mu_{2}(x), \mu_{4}(x)\right\} \\
& \text { Minimize } \mathrm{w}_{1}\left\{v_{0}(x), v_{2}(x), v_{4}(x)\right\} \\
& \text { Minimize } \mathrm{w}_{2}\left\{\pi_{0}(x), \pi_{2}(x), \pi_{4}(x)\right\} \\
& \text { Subject to constraints } \\
& \mu_{k}(x) \geq v_{k}(x), k=0,2,4 \\
& v_{k}(x) \geq 0, k=0,2,4 \\
& \mu_{k}(x)+v_{k}(x) \leq 1, k=0,2,4 \\
& x 11+\mathrm{x} 21+\mathrm{x} 31=200, \\
& \mathrm{x} 13+\mathrm{x} 23+\mathrm{x} 33=100, \\
& \mathrm{x} 11+\mathrm{x} 12+\mathrm{x} 13+\mathrm{x} 14=400, \\
& \mathrm{x} 21+\mathrm{x} 22+\mathrm{x} 23+\mathrm{x} 24=150, \\
& \mathrm{x} 31+\mathrm{x} 32+\mathrm{x} 33+\mathrm{x} 34=300, \\
& \mathrm{xij} \geq 0, \mathrm{i}=1,2,3, \mathrm{j}=1,2,3,4,
\end{aligned}
$$

where $\pi_{0}(x)=1-\mu_{0}(x)-v_{0}(x) \quad \pi_{2}(x)=1-\mu_{2}(x)-v_{2}(x) \quad \pi_{4}(x)=1-\mu_{4}(x)-v_{4}(x)$ and $w_{1}, w_{2}$ are any pre assigned (such that $\mathrm{w}_{1}+\mathrm{w}_{1}+\mathrm{w}_{2}=1$ ) weights.

Next, we know that if in an optimization problem $\mathrm{G}$ denotes goal and $\mathrm{C}$ denotes constraints, we have the decision $\mathrm{D}$ defined by $D=G \cap C=\left\{\left\langle x, \mu_{G}(x) \cap \mu_{C}(x), v_{G}(x) \cup v_{C}(x)>: x \in R^{n}\right\}\right.$. This operator can be easily generalized and applied to the IFO problem:

$$
D=\left\{\left\langle x, \mu_{D}(x), v_{D}(x), \pi_{D}(x)\right)>: x \in R^{n} \text { and } \pi_{D}(x)=\left[1-\left(\mu_{D}(x)+v_{D}(x)\right)\right]\right\}, \mu_{D}=\bigcap_{i=1}^{p+q} \mu_{i}, v_{D}=\bigcup_{i=1}^{p+q} v_{i}
$$

Hence $\mu_{D}=\min _{i=1}^{p+q} \mu_{i}, x \in R^{n}, \mu_{D} \leq \mu_{i}$ and $v_{D}=\max _{i=1}^{p+q} v_{i}, x \in R^{n}, v_{D} \geq v_{i}$. So it can be transformed to the following system of equations:

$$
\begin{aligned}
& \alpha \leq \mu_{i}(x), i=1,2, \ldots,(p+q), \\
& \beta \geq v_{i}(x), i=1,2, \ldots,(p+q), \\
& \alpha \geq \beta, \beta \geq 0, \alpha+\beta \leq 1,
\end{aligned}
$$

where $\alpha$ denotes the minimal acceptable degree of objective(s) and constraints and $\beta$ denotes the maximal degree of rejection of objective(s) and constraints. Now the IFO problem can be transformed to the following crisp (non-fuzzy) optimization problem which can be easily solved numerically or by using any standard software:

$$
\text { Maximize } w_{1} \alpha-w_{1} \beta-w_{2}(1-\alpha-\beta)
$$

Subject to the constraints

$$
\begin{aligned}
& \alpha \leq \mu_{i}(x), i=1,2, \ldots,(p+q), \\
& \beta \geq v_{i}(x), i=1,2, \ldots,(p+q), \\
& \alpha \geq \beta, \beta \geq 0, \alpha \geq 0, \alpha+\beta \leq 1,
\end{aligned}
$$

and crisp constraints and $w_{1}+w_{1}+w_{2}=1$.

So, in our case, if our primary aim is to minimize the degree of hesitation in the optimum solution, the following intuitionistic fuzzy optimization problem may be considered

Maximize $\mathrm{Z}=.1 * \alpha-.1 * \beta-.8 *(1-\alpha-\beta)$

Subject to the constraints

$x 11+x 21+x 31=200$,

$x 13+x 23+x 33=100$, 


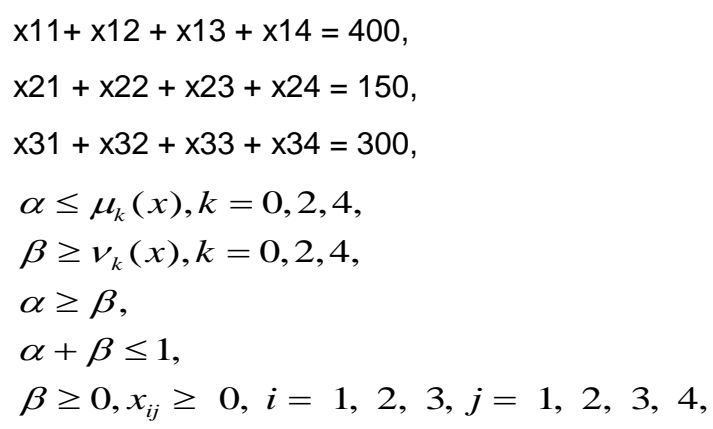

It can be solved by using LINGO (Version 13.0.2.16) software and the solution satisfies the objective with degree of acceptance: 0.34 and degree of rejection: 0.34 and costs $\$ 2,330,000$, which is same as the analogous fuzzy linear programming problem. Here, the degree of hesitation is 0.32 . The solution table is as follows:

Table 2. Solution (using LINGO) of IF optimization problem

\begin{tabular}{|c|c|c|c|c|c|}
\hline $\begin{array}{c}\text { Optimum Table } \\
(\mathbf{H})\end{array}$ & Market 1 & Market 2 & Market 3 & Market 4 & Capacity \\
\hline Port 1 & 0 & 0 & 36.05 & 363.95 & 400 \\
\hline Port 2 & 150 & 0 & 0 & 0 & 150 \\
\hline Port 3 & 50 & 186.05 & 63.95 & 0 & 350 \\
\hline Demand & 200 & 200 & 100 & 350 & \\
\hline
\end{tabular}

Again in some cases, it may happen that the main target is to minimize the degree of rejection of the optimum solution. In that case, the intuitionistic fuzzy optimization problem may take the following form:

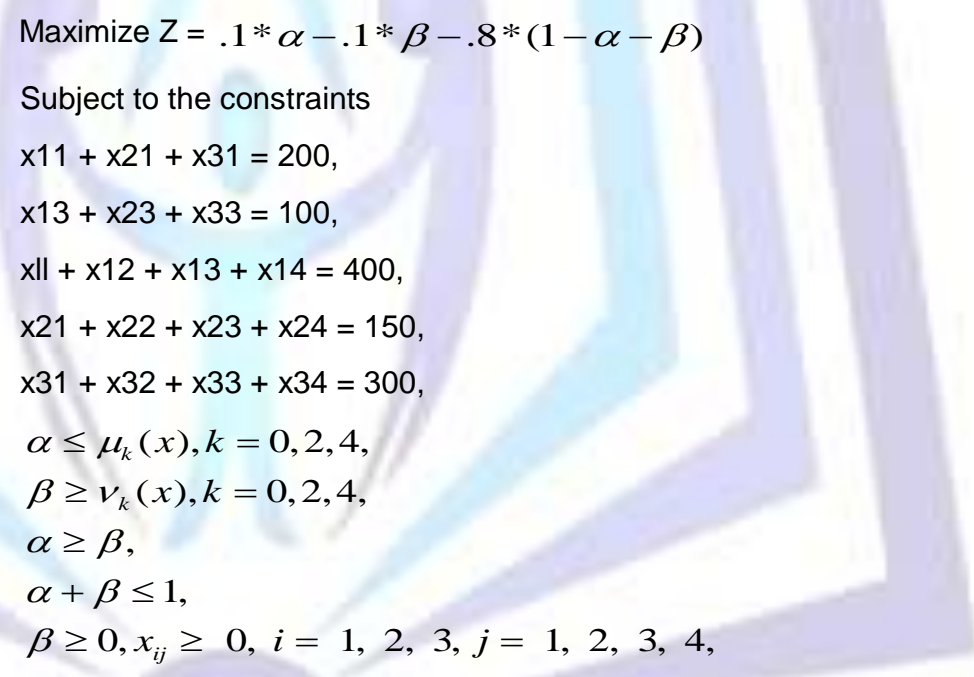

It can also be solved using LINGO (Version 13.0.2.16) software and the solution satisfies the objective with degree of acceptance: 0.14 and degree of rejection: 0.02 and costs $\$ 2,428,571$. In this case, the solution table is as follows:

Table 3. Solution while minimizing degree of rejection

\begin{tabular}{|c|c|c|c|c|c|}
\hline $\begin{array}{c}\text { Optimum Table } \\
\text { (R) }\end{array}$ & Market 1 & Market 2 & Market 3 & Market 4 & Capacity \\
\hline Port 1 & 20.65 & 0 & 30.93 & 348.42 & 400 \\
\hline Port 2 & 150 & 0 & 0 & 0 & 150 \\
\hline Port 3 & 29.34 & 201.58 & 69.08 & 0 & 350 \\
\hline Demand & 200 & 200 & 100 & 350 & \\
\hline
\end{tabular}

\section{CONCLUSION}

It is easy to observe that the cost comes down when our primary aim is to reduce the degree of hesitation. But, if we target to reduce the degree of rejection, the cost goes up. We can give different weights to each of degree of acceptance, degree of rejection as well as degree of hesitation. In each circumstances, an intuitionistic fuzzy optimization problem surfaces 
and so the optimum solution becomes different. Actually, a new concept to the optimization problem in an IF environment is used in this paper. It allows using the degree of hesitation which cannot always be ignored in case of intuitionistic fuzzy set. Finally, it may be noted that customer (in our case, decision maker) is our king and his (or her!) choice is final. Hence there is no standard form of an intuitionistic fuzzy optimization problem and it can be customized according to requirement always. Our effort is on to find a concrete and everywhere-accepted format of intuitionistic fuzzy optimization problem and a standard solution procedure.

\section{REFERENCES}

[1] Angelov P. Plamen. Optimization in an intuitionistic fuzzy environment, Fuzzy Sets and Systems, Vol. 86, 1997, 299306.

[2] Atanassov K. Intuitionistic fuzzy sets, Fuzzy Sets and Systems, Vol. 20, 1986, 87-96.

[3] Atanassov, K. Ideas for intuitionistic fuzzy sets equations, inequalities and optimization, Notes on Intuitionistic Fuzzy Sets, Vol. 1, 1995, No. 1, 17-24.

[4] Atanassov, K. Two theorems for intuitionistic fuzzy sets, Fuzzy Sets and Systems, Vol. 110, 2000, $267-269$.

[5] Bellman, R. E., L. A. Zadeh. Decision making in a fuzzy environment, Management Science, Vol. 17, 1970, B141B164.

[6] Roy, Kr. Tapan, Banerjee, S. Solution of Single and Multi objective Stochastic Inventory Models with Fuzzy Cost Components by Intuitionistic Fuzzy Optimization Technique, Advances in Operation Research, Vol. 2010(2010), Article ID 765278.

[7] Szmidt, E., J. Kacprzyk. Distances between intuitionistic fuzzy sets, Fuzzy Sets and Systems, Vol. 114, 1997, 505518.

[8] Wikipedia Contributors. Intuitionism. Wikipedia, The Free Encyclopedia. [22 Dec., 2012 10:10 UTC] http://en.wikipedia.org/wiki/Intuitionism (accessed 22 Dec., 2012).

[9] Garai, A., Roy, Kr. T. Weighted intuitionistic fuzzy Delphi method, Journal of Global Research in Computer Science, Vol. 4(7), 2013.

\section{Author' biography with Photo}

\section{Arindam Garai}

Me@ passionately love to mentor using innovation, mixing positive attitude and enthusiasm of a born leader and with strong desire to be a part of success in my chosen academic life.

Books, especially on self-help, mystery are my first love. Confidence and mental power have been gained from those printed words.

Chess is my favourite game. It has improved my ability to think logically.

Research Area: Fuzzy Set, Intuitionistic Fuzzy Optimization, Fuzzy and Intuitionistic Fuzzy Decision Making, Delphi Method etc.

Mail fuzzy_arindam@yahoo.com

Mob.+91-9932890115

$$
\text { Dr. Tapan Kumar Roy }
$$

Me@ guide my students professionally as well as personally.

Research Area: Fuzzy and Intuitionistic Fuzzy set Theory, Inventory, Transportation, Reliability Optimization, Portfolio Optimization, Fuzzy and Stochastic Optimization etc.

Mail roy_t_k@yahoo.co.in

Mob.+91-9477419380 